\title{
Interactive effects of genotype and food quality on consumer growth rate and elemental content
}

\author{
Clay Prater, ${ }^{1,3}$ Nicole D. Wagner, ${ }^{1}$ and Paul C. Frost ${ }^{2}$ \\ ${ }^{1}$ Environmental and Life Science Graduate Program, Trent University, Peterborough, Ontario K9J 7 B8 Canada \\ ${ }^{2}$ Department of Biology, Trent University, Peterborough, Ontario K9L 128 Canada
}

\begin{abstract}
Consumer body stoichiometry is a key trait that links organismal physiology to population and ecosystem-level dynamics. However, as elemental composition has traditionally been considered to be constrained within a species, the ecological and evolutionary factors shaping consumer elemental composition have not been clearly resolved. To this end, we examined the causes and extent of variation in the body phosphorus $(\mathrm{P})$ content and the expression of P-linked traits, mass specific growth rate (MSGR), and P use efficiency (PUE) of the keystone aquatic consumer Daphnia using lake surveys and common garden experiments. While daphnid body $\% \mathrm{P}$ was relatively constrained in field assemblages sampled across an environmental $\mathrm{P}$ gradient, unique genotypes isolated from these lakes showed highly variable phenotypic responses when raised across dietary P gradients in the laboratory. Specifically, we observed substantial inter- and intra-specific variation and differences in daphnid responses within and among our study lakes. While variation in Daphnia body \% $\mathrm{P}$ was mostly due to plastic phenotypic changes, we documented considerable genetic differences in daphnid MSGR and PUE, and relationships between MSGR and body P content were highly variable among genotypes. Overall, our study found that consumer responses to food quality may differ considerably among genotypes and that relationships between organismal life-history traits and body stoichiometry may be strongly influenced by genetic and environmental variation in natural assemblages.
\end{abstract}

Key words: Daphnia; ecological stoichiometry; $G \times E$ interaction; life-history evolution; phenotypic plasticity; phosphorus use efficiency; reaction norm.

\section{INTRODUCTION}

Consumers can strongly affect ecosystem-level dynamics through the expression of phenotypic traits that influence ecological interactions and alter material fluxes through the environment (Miner et al. 2005, Matthews et al. 2011, Declerck et al. 2015, Gibert et al. 2015). One trait that is particularly important in this context is body elemental composition, which ranges widely among taxa and across trophic levels (Frost et al. 2006, Persson et al. 2010, El-Sabaawi et al. 2014, Jeyasingh et al. 2014). In general, metazoan consumers appear to maintain a more constant elemental composition and have a higher nutrient content than their food resources (Andersen and Hessen 1991, Hood and Sterner 2010, Persson et al. 2010). This creates stoichiometric imbalances, which cause consumers to make physiological adjustments (e.g., altered nutrient uptake and excretion) to regulate their body elemental composition (Frost et al. 2005). However, there is accumulating evidence that consumer responses to dietary elemental limitation may result in significant variation in life-history and body stoichiometry

Manuscript received 9 May 2016; revised 7 February 2017; accepted 21 February 2017. Corresponding Editor: Carla E. Cáceres.

${ }^{3}$ E-mail: prater.clay@gmail.com even among closely related taxa (DeMott et al. 2004, Weider et al. 2008, Frisch et al. 2014). Here, we investigate the origin and extent of these differences by examining variation in the body phosphorus $(\mathrm{P})$ content and expression of P-linked traits in field-caught genotypes of the aquatic consumer, Daphnia.

Due to its central role in influencing elemental dynamics in aquatic food webs, stoichiometric variation in Daphnia has been studied extensively. This work has documented large interspecific differences in Daphnia body \%P ( 0.8-1.9\%P; DeMott and Pape 2005, Hood and Sterner 2010), which have generally been attributed to adaptations resulting from natural selection acting on life-history and physiological traits (Elser et al. 1996, Elser 2006, Jeyasingh et al. 2014). For example, because rapid growth requires considerable amounts of $\mathrm{P}$ to be invested in ribosomal RNA synthesis, evolution of fast growth rates appears to be linked to higher Daphnia body $\%$ P (i.e., the growth rate hypothesis; Elser et al. 2003). Laboratory-based studies have supported this indirect mode of stoichiometric evolution by identifying correlations between key traits such as size at maturity, fecundity, growth, P use efficiency (PUE), and daphnid body P content (Gorokhova et al. 2002, Weider et al. 2008, Jeyasingh et al. 2009, Frisch et al. 2014). Differences in the expression of P-linked traits under dietary P-limitation have also been documented in animals evolving 
under different food quality conditions (DeMott et al. 2004, Frisch et al. 2014), further suggesting that integrated changes in Daphnia P metabolism/content may be adaptive in field environments. However, despite this progress, a mechanistic understanding of how evolutionary processes shape the elemental composition of natural assemblages remains elusive. This is partly because organismal body stoichiometry reflects both heritable and non-heritable trait responses that may be influenced by a variety of environmental factors in addition to food quality. Variation can also arise at many different biological scales including within a single clonal genotype, among genetically distinct clones within a species, and from differences between species (DeMott et al. 2004, Hood and Sterner 2010, Frisch et al. 2014). Thus, to better understand how these factors shape consumer elemental composition in nature, there is a clear need for developing a framework for distinguishing between genetic and ecological sources of variation.

Organismal trait expression depends on interactions between an individual's genotype and the environment (Stearns 1992, Pigliucci 2005). Although it is difficult to separate and study the relative influence of consumer genetics and food quality on daphnid body P content in the field, common garden experiments provide a means of isolating such effects under controlled laboratory conditions (Pigliucci 2001, Kawecki and Ebert 2004). Using this approach, unique daphnid clones can be collected from a lake, and reaction norms for key stoichiometrically relevant traits (body $\% \mathrm{P}$, growth, and PUE) can be characterized across dietary P supply gradients. Differences in the mean expression of each trait within and among diet treatments may then be attributed either to genetic variation or phenotypic plasticity, respectively through simple variance partitioning of main effects using an analysis of variance (ANOVA) framework (Pigliucci 2001). Heritability of trait plasticity can also be quantified by identifying trait variation caused by genotype by diet $(G \times D)$ interactions by comparing the shapes of consumer reaction norms across food $\mathrm{P}$ gradients. This type of analysis provides information on the extent of intra-specific trait variation, how trait means and variance might change with resource supply, and whether or not these responses could lead to trait evolution in a given lake (Stearns 1992, Schlichting and Pigliucci 1998). Further, by extending these techniques to include additional species collected from contrasting resource environments, we can compare species responses to elemental limitation and examine how these differences influence species distributions in nature. In this way, we can combine principles from ecological stoichiometry and life-history theory to provide powerful insights into how evolutionary processes shape consumer elemental variation and gain a better appreciation of the ecological consequences of these changes.

In this study, we examined sources of variation in three phenotypic traits: MSGR, body $\mathrm{P}$ content, and PUE among multiple clones in two separate Daphnia species. We first measured differences in daphnid body $\%$ $\mathrm{P}$ of field-caught animals from nine lakes located in two distinct ecoregions differing in lake $\mathrm{P}$ concentrations. Then, we raised isolated genotypes from these lakes in common garden environments where we manipulated their dietary $\mathrm{P}$ content and quantified changes in their trait expression. Following these experiments, we compared the relative extent of inter- vs. intraspecific trait variation. Next, we contrasted responses of our experimental genotypes to estimate levels of variation within lakes and to test for evidence of differences in trait responses among lakes. Finally, we investigated possible mechanisms influencing trait expression and evolution in these lakes by comparing the relative effects of genotype, diet, and $\mathrm{G} \times \mathrm{D}$ interactions on daphnid trait variation and by examining relationships between MSGR and body $\% \mathrm{P}$ across food quality gradients.

\section{Methods \\ Study sites}

We sampled lakes that were located within a narrow $(30 \mathrm{~km})$ radius spanning two distinct ecoregions in south central Ontario, Canada. One subset of our lakes $(n=5)$ were in the Kawartha Highlands, which is located on the southern boundary of the Precambrian Shield. Land use in the area is mostly forested, and lakes have relatively fewer residences on their shorelines (Hicks and Frost 2011). The other group of study lakes were in the Kawartha Lakes region $(n=4)$ located south of the Highlands and the Canadian Shield. Geology around these lakes is mostly comprised of limestone and dolomite, and land use in the area is $\sim 50 \%$ cropland with high residential development along the shores of the lakes (Crins et al. 2009). We chose study lakes that spanned a total phosphorus (TP) gradient from $4-20 \mu \mathrm{g} / \mathrm{L}$ to capture a range of $\mathrm{P}$ supply across study lakes.

\section{Lake water and Daphnia collection and processing}

We sampled lakes in early spring to estimate withinand among-lake variability in TP and daphnid body $\% \mathrm{P}$. Water column TP samples were collected at the lake surface $(\sim 0.5 \mathrm{~m})$ and $1 \mathrm{~m}$ above the bottom of the lake using a Van Dorn sampler. Daphnia samples were collected by taking vertical tows in the deepest part of each lake with an $80-\mu \mathrm{m}$ zooplankton net. Samples were brought back to the laboratory on ice for processing. An unfiltered water sample was used to estimate TP using a persulfate digestion followed by molybdate-blue ascorbic acid colorimetry and spectroscopy (Greenberg et al. 1992). Daphnia samples were live sorted, rinsed, and random subsets of 5-10 individuals were saved separately in aluminum cups and dried at $60^{\circ} \mathrm{C}$ for at least $24 \mathrm{~h}$. These animals were weighed using a microbalance, and daphnid body $\mathrm{P}$ content was determined $(n=5)$ for animals from each lake using methods described for TP. 


\section{Daphnia isolation for common garden experiments}

Daphnia collected in the field were isolated during the spring of 2012 and subsequently used in laboratory growth experiments. From each lake, we randomly selected 25 individuals and placed each into an individual tube containing $40 \mathrm{~mL}$ of artificial COMBO media (Kilham et al. 1998). These daphnids were fed high quality diets (C:P 80-100) of Scenedesmus obliquus (Canadian Phycological Culture Centre strains 10) ad libitum. To establish separate clonal lines, a single parthenogenetically produced neonate $(<24 \mathrm{~h}$ old from second or third broods) from each of these mother Daphnia was removed and separated into its own tube to completely isolate distinct genotypes. These first-generation individuals were raised until reproduction, and offspring from their second or third broods were used to establish multi-animal cultures in $400-\mathrm{mL}$ beakers. We used these clonal sister populations to produce large numbers of third- to fifthgeneration neonates for each unique genotype, which we used for common garden growth experiments. These methods were specifically aimed to greatly reduce or eliminate maternal effects from field conditions and to avoid reducing variation in daphnid phenotypic differences due to acclimation to lab conditions. Due to high mortality rates during this period (occasionally $<20 \%$ survival), between three and five genetically distinct Daphnia clonal lines were isolated from the initial fieldcaught mothers collected from each lake. Thus, it is possible that our variation estimates were biased by selecting for genotypes that were most capable of growing and reproducing in laboratory environments. In all, a total of 36 unique genotypes (see Daphnia genotyping) were used in growth experiments. However, to ensure that differences in daphnid phenotypic responses were not due to random error we replicated growth experiments by conducting two trials on a subset of random genotypes $(n=4)$, for a total of 44 separate trials.

\section{Dietary P manipulation and growth experiments}

Algal food was grown in artificial growth media (Sterner et al. 1993) under high light conditions. We manipulated algal $\mathrm{C}: \mathrm{P}$ by altering daily dilution rates and media $\mathrm{P}$ concentrations in batch cultures (two to three separate flasks) grown at three different $\mathrm{P}$ concentrations $(\sim \mathrm{C}: \mathrm{P}$ 80, C:P 500, and C:P 1000). We estimated the nutrient content of algal cultures by filtering algae from each treatment onto $0.7-\mu \mathrm{m}$ ashed glass fiber filters. We then measured algal $\% \mathrm{P}$ using methods described in Lake water and Daphnia collection and processing, and algal \% $\mathrm{C}$ content was determined using an Elemental Analyzer (Vario EL III, Elementar Incorporated, Mt. Laurel, New Jersey, USA). Next, we used these estimates to mix nominal diet treatments for experiments. Additional filters were saved to determine the final elemental composition of mixed food that was provided to experimental animals. Post-mixed diets were relatively stable (C:P molar ratios $=580 \pm 29.8[$ mean $\pm \mathrm{SD}$ ], $292 \pm 26.5$, and $90 \pm 8.4$ ) and closely matched our target $\mathrm{C}: \mathrm{P}$ ratios across all experiments.

Animals were fed high quantities [4 mg C/L, every other day] of algae spanning a dietary $\mathrm{C}: \mathrm{P}$ gradient: low $\mathrm{P}(\sim \mathrm{C}: \mathrm{P} 580)$, middle $\mathrm{P}(\sim \mathrm{C}: \mathrm{P} 292)$, and high $\mathrm{P}(\sim \mathrm{C}: \mathrm{P} 90)$, which matches the range of $\mathrm{P}$ content typically observed in natural seston (Elser et al. 2000a) and is known to produce moderate to mild $\mathrm{P}$ limitation in the higher $\mathrm{C}: \mathrm{P}$ treatments, respectively (Acharya et al. 2004, Wagner and Frost 2012). Approximately (60) neonates from broods 2-5 for each genotype were rinsed with $\mathrm{P}$-free combo media and placed separately into $20-\mathrm{mL}$ vials. Initial neonate mass ( $n=3$ of 20 pooled individuals) of each genotype was also estimated from dried samples and used for subsequent MSGR calculations. After $6 \mathrm{~d}$, groups of five animals were saved from each diet treatment for $\mathrm{P}$ content analysis $(n=5$ replicates). Individual animals were also dried and weighed to determine their final body mass. We calculated MSGR by subtracting the natural $\log$ of the initial neonate mass from the natural $\log$ of the final average individual mass and dividing by the duration of the experiment (Tessier and Goulden 1987). Daphnid PUE was determined by taking the ratio of MSGR to body $\% \mathrm{P}$, which differs qualitatively from other non mass-specific estimates (Jeyasingh et al. 2009, Frisch et al. 2014) but was necessary to control for large differences in initial neonate mass between species. Finally, ten individuals from each experimental genotype were pooled, frozen, and stored in a $-80^{\circ} \mathrm{C}$ freezer for genotyping.

\section{Daphnia genotyping}

Each experimental genotype was first identified to species complex (Daphnia mendotae or Daphnia pulex) using image-based keys (Haney et al. 2013), and we confirmed these identifications by sequencing the $12 \mathrm{~S}$ rDNA gene (see Appendix S1: Supplementary Methods). To determine species identity, we conducted allozyme electrophoresis analyses using the enzymes AO (EC 1.2.3.1) to distinguish between $D$. dentifera and $D$. mendotae and LDH (EC 1.1.1.27) to separate D. pulex and D. pulicaria (Hebert and Beaton 1993). All genotypes were determined to be either D. pulicaria $(n=25)$ or $D$. mendotae $(n=11)$, and we subsequently used four additional polymorphic loci: ME (1.1.1.40), MDH (EC 1.1.1.37), GPI (EC 5.3.1.9), and PGM (EC 5.4.2.2) to identify genetically distinct clones.

\section{Statistical analyses}

Prior to parametric analyses, all variables were tested for normality and homogeneity of variances using Shapiro-Wilk and Levene's test, respectively. To improve homoscedasticity, MSGR and PUE were ln-transformed. We compared differences in daphnid body $\% \mathrm{P}$ between regions and among sites, using a two-way analysis of 
variance (ANOVA). Next, we examined relationships between Daphnia body P content and water column TP in study lakes using an ordinary least squares regression. Before conducting the main analyses for the common garden experiments, we analyzed duplicated growth experiments $(n=8)$ to ensure that clonal responses could be replicated across multiple trials. To compare clonal growth responses between trials, we conducted a homogeneity of slopes test in SMATR (Falster et al. 2006) and found no significant differences between experimental trials for any clone $(P>0.05$, Appendix S1: Table S1) indicating that experimental artifacts on clonal responses were negligible. We subsequently excluded one of the replicated trials for each clone from the main analysis to avoid repeated measures.

For common garden experiments, we first examined intra- and interspecific differences in MSGR, body $\% \mathrm{P}$, and PUE using two-way ANOVAs and compared the extent of variation within and between species through variance components analyses using random effects general linear models where denominators of $F$-ratio tests were corrected according to Littell et al. (2002). These models were fit using restricted maximum likelihood estimates (REML), which provide unbiased estimators of variance components for unbalanced experimental designs (Searle et al. 1992). We also compared mean trait differences between species using least squared means estimates. Next, we conducted two-way mixedeffects ANOVAs with interaction to examine whether Daphnia trait expression varied within/among lakes, across dietary $\mathrm{P}$ gradients, and to test for differences in trait responses among lakes indicated by lake $\times$ diet ( $\mathrm{L}$ $\times$ D) interactions. For these models, Genotype (Lake) terms were treated as random, and all other terms were considered fixed. We then preformed variance components analyses using corrected random effects models for each species individually to compare the relative strength of genotype, diet, and $\mathrm{G} \times \mathrm{D}$ interactions on these responses. Finally, we used an ordinary least squares regression to determine how diet influenced relationships between daphnid MSGR and body \%P. All statistics were conducted using SAS (Version 9.4; SAS Institute, Cary, North Carolina, USA).

\section{RESULTS}

\section{Daphnia body P content from source lakes and regional species differences}

Mean daphnid body \% $\mathrm{P}$ in lake assemblages fell within a relatively narrow range $(1.22-1.41 \% \mathrm{P})$. While daphnid body $\mathrm{P}$ content varied among lakes, it did not differ significantly between regions (Fig. 1A) despite contrasting lake water column TP concentrations. Differences in assemblage $\mathrm{P}$ content were mostly due to intraspecific variation as we found little overlap in species distributions between regions (Fig. 1B). Specifically, clones collected in lower nutrient Highlands Lakes were
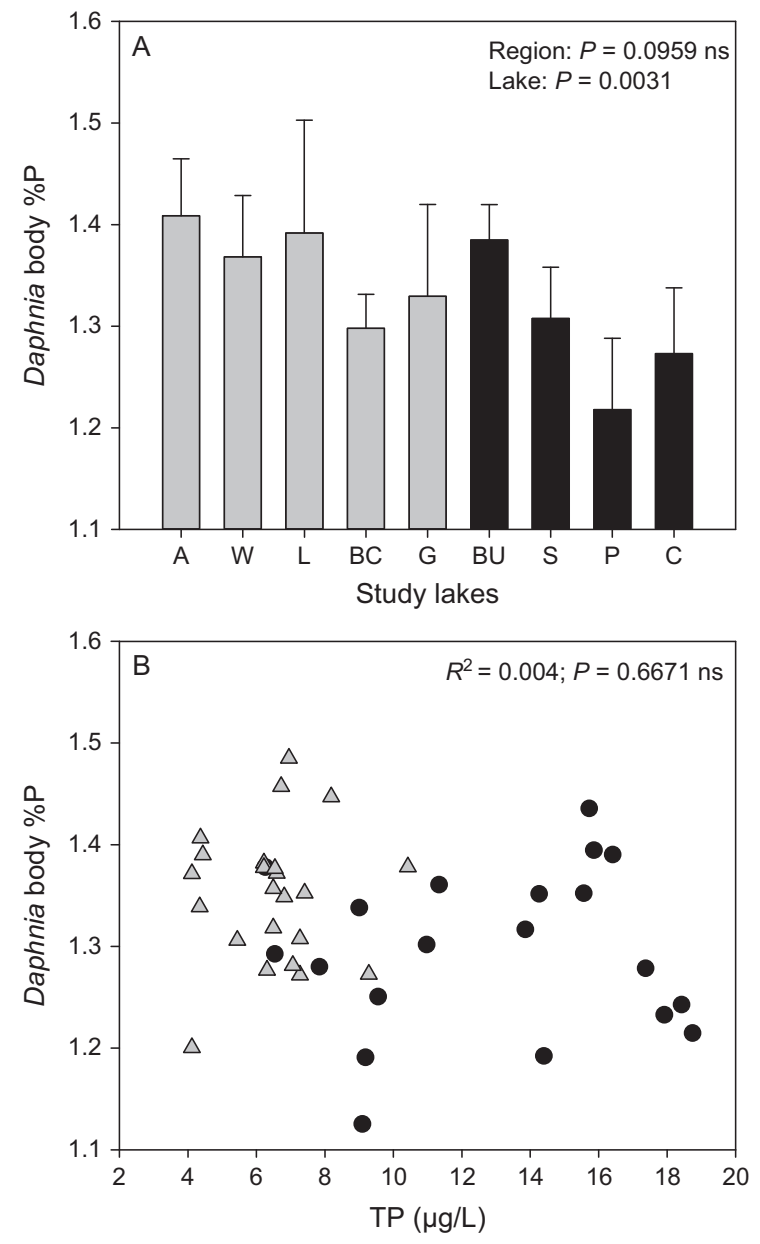

FIG. 1. Daphnia body phosphorus content $(\% \mathrm{P})$ in study lakes. (A) Differences between regions and among lakes were determined using a two-way ANOVA. Highlands Lakes Anstruther (A), Wolf (W), Looncall (L), Big Cedar (BC), and Gold $(\mathrm{G})$ are shown as gray bars and Kawartha Lakes Buckhorn (BU), Stoney (S), Pigeon (P), and Chemong (C) are shown as black bars. (B) Relationships between daphnid body $\% \mathrm{P}$ and water column total $\mathrm{P}$ were estimated using an ordinary least squares regression. Daphnia pulicaria are represented as gray triangles and $D$. mendotae are shown as black circles. $P$-values are given for ANOVAs, and an $R^{2}$ and $P$-value are reported for the linear regression. Non-significant effects have "ns" indicated for each analysis.

all identified as D. pulicaria. Only three clones of this species were found in Kawartha Lakes, which were instead mostly populated by $D$. mendotae.

\section{Daphnia responses to dietary P availability}

Compared to field assemblages, we found a large amount of trait variation among isolated genotypes raised in common garden experiments. Inter-specific responses to dietary $\mathrm{P}$ differed strongly as $D$. pulicaria clones displayed higher MSGR and PUE, whereas D. mendotae had greater body P content overall (Table 1, 
TABLE 1. Trait differences and variation within and among Daphnia species.

\begin{tabular}{lccccccc}
\hline \hline Trait & Source & $d f$ & SS & MS & $F$ & $P$-value & $\%$ Var \\
\hline MSGR & Species & 1 & 0.536 & 0.536 & 9.11 & 0.0048 & 17.11 \\
& Clone(Species) & 34 & 2.000 & 0.059 & 17.65 & $<0.0001$ & 29.87 \\
& Total Model & 35 & 2.535 & 0.072 & 21.73 & $<0.0001$ & 46.97 \\
& Error & 1020 & 3.400 & 0.003 & & & 53.03 \\
$\%$ PP & Species & 1 & 1.119 & 1.119 & 12.20 & 0.0013 & 12.5 \\
& Clone(Species) & 34 & 3.119 & 0.092 & 2.58 & $<0.0001$ & 9.32 \\
& Total Model & 35 & 4.228 & 0.121 & 3.40 & $<0.0001$ & 21.82 \\
& Error & 440 & 15.645 & 0.036 & & & 78.18 \\
PUE & Species & 1 & 0.250 & 0.250 & 14.49 & 0.0006 & 34.57 \\
& Clone(Species) & 34 & 0.587 & 0.017 & 14.16 & $<0.0001$ & 32.8 \\
& Total Model & 35 & 0.867 & 0.025 & 20.33 & $<0.0001$ & 67.38 \\
& Error & 440 & 0.536 & 0.001 & & 32.62
\end{tabular}

Notes: Degrees of freedom $(d f)$, type III sum of squares (SS), mean squares (MS), $F$-ratio of mean squares $(F)$, and $P$-values are reported for ANOVAs examining differences in daphnid mass specific growth rate (MSGR), body phosphorus content (\%P), and $\mathrm{P}$ use efficiency (PUE). The proportion of trait variation (\%Var) explained by each factor is also reported from variance component analyses. Species trait means for Daphnia pulicaria are MSGR 0.377, \%P 1.385, PUE 0.275 , and for D. mendotae are MSGR 0.317, \%P 1.502, PUE 0.217. Post hoc analyses indicate significant differences in trait means between species $(P<0.0001)$.

Fig. 2). However, intraspecific variation in body $\% \mathrm{P}$ and PUE was nearly as extensive as differences between species and was nearly two times greater for MSGR (Table 1). Significant within- and among-lake differences in trait expression of field-caught genotypes suggests that organisms could vary considerably in their responses to dietary P-limitation in nature (Table 2). Furthermore, we also detected significant $\mathrm{L} \times \mathrm{D}$ interactions for all traits providing evidence that lake assemblages differed in their responses to dietary $\mathrm{P}$ supply.

\section{Genotype, diet, and $G \times D$ effects}

Genotype and diet strongly affected daphnid phenotypic trait expression, and the influence of these factors was qualitatively similar between species explaining a large proportion of overall trait variation (66-82\%). In general, trait expression was highly variable differing widely among genotypes within a diet treatment and across the dietary gradient (Table 3, Fig. 2). Significant main effects were found for all traits except for genotypic differences in $D$. mendotae $\% \mathrm{P}$ and dietary effects on PUE (Table 3). We also found significant $G \times D$ interactions for MSGR and PUE but no evidence of interactive effects on daphnid body $\mathrm{P}$ content in either species. The relative strength of plastic vs. genetic variation differed across traits as variation in daphnid body $\% \mathrm{P}$ was mostly affected by dietary $\mathrm{P}$ content, whereas genetic and $\mathrm{G} \times \mathrm{D}$ interactions generally had greater effects on MSGR and PUE. Overall, these changes in trait expression resulted in weak positive relationships between daphnid MSGR and body $\mathrm{P}$ content for both species (Fig. 3).

\section{Discussion}

Despite originating from lake assemblages showing relatively limited variability in Daphnia body $\mathrm{P}$ content at the time of sampling, we documented considerable variation in MSGR, body $\% \mathrm{P}$, and PUE when animals were grown across dietary $\mathrm{P}$ gradients. These responses were largely nonlinear, and trait variation was high both within a species and between species. We also observed significant trait variation among genotypes collected from within the same lake and found differential responses of animals collected across lakes. Trait expression was significantly affected by genotype, diet, and $G$ $\times \mathrm{D}$ interactions, meaning that both genetic variation and plastic phenotypic responses affected daphnid lifehistory and body $\mathrm{P}$ composition in our study.

Daphnid body $\% \mathrm{P}$ differed significantly among field assemblages but overlapped between regions. Overall variation in our study lakes was relatively low and intermediate compared to results from field collections documenting both minimal variation in daphnid stoichiometry (Andersen and Hessen 1991) and widely differing body $\mathrm{P}$ content in natural assemblages (DeMott et al. 2004). Contrary to previous work, we also found weaker evidence of regional differences in body $\% \mathrm{P}$ (Elser et al. 2000b). These contrasting results are perhaps not surprising and could be due to differences in spatial scales, species examined, and the degree of nutrient limitation between these studies. Moreover, our field collections were not designed to measure the full extent of body $\% \mathrm{P}$ variation among our field assemblages but instead focused on characterizing differences in $\mathrm{P}$ supplies among lakes and between regions. Daphnia body $\mathrm{P}$ was not related to lake TP and was likely constrained by the relatively high and consistent food quality conditions $(\mathrm{C}: \mathrm{P}=152 \pm 61)$ during the early spring sampling period. Although we detected only minor differences in body $\% \mathrm{P}$ among lakes, Daphnia body $\mathrm{P}$ content and P-linked trait expression varied greatly in isolated genotypes raised in common garden environments. 

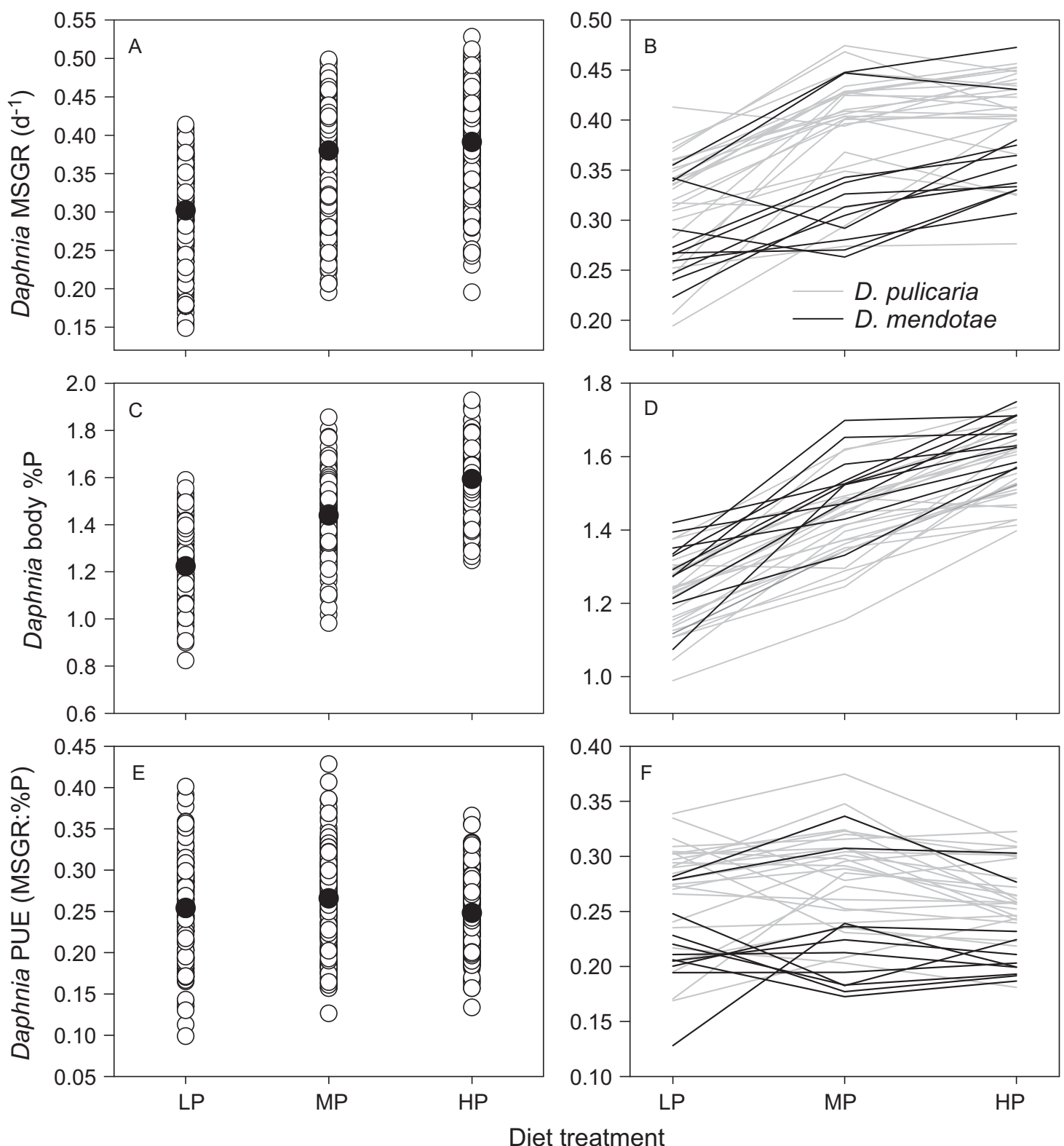

FIG. 2. Trait means with variance and reaction norm plots showing variation in consumer phenotypic response across dietary nutrient gradients. Changes in mass-specific growth rate (A and B: MSGR), body phosphorus content (C and D: \% P), and P use efficiency (E and F: PUE) are shown across low P (LP), middle P (MP), and high P (HP) diets.

We found substantial taxonomic variation in daphnid responses to dietary $\mathrm{P}$ supply. As in earlier studies, each species displayed significant differences in growth and body $\% \mathrm{P}$ when raised in different nutrient environments (Elser et al. 2000b, Acharya et al. 2004, Seidendorf et al. 2010, Hood and Sterner 2014). However, we also observed a comparable amount of intraspecific variation in both traits, and body $\mathrm{P}$ content in our study animals spanned nearly the entire range of all previously documented daphnid species. While large differences in body $\% \mathrm{P}$ have been reported for
D. pulicaria growing across laboratory dietary $\mathrm{P}$ gradients, $D$. mendotae has previously been identified as a relatively invariant taxon (DeMott and Pape 2005, Hood and Sterner 2010). In contrast, we found comparable amounts of variation in body $\mathrm{P}$ content in this taxon (see DeMott et al. 2004) along with extensive variation in MSGR and PUE in both species. These observations indicate that consumer elemental variation might be more common than previously acknowledged by stoichiometric theory (Jeyasingh et al. 2014) and could represent a form of adaptive phenotypic 
TABLE 2. Trait differences in field-caught Daphnia within/ among lakes, across dietary phosphorus $(\mathrm{P})$ gradients, and due to Lake $\times$ Diet interactions.

\begin{tabular}{|c|c|c|c|c|c|}
\hline Trait and Source & $d f$ & SS & MS & $F$ & $P$-value \\
\hline \multicolumn{6}{|l|}{ MSGR } \\
\hline$\overline{\text { Lake }}$ & 8 & 1.509 & 0.189 & 106.05 & $<0.0001$ \\
\hline Genotype (lake) & 27 & 1.002 & 0.037 & 20.87 & $<0.0001$ \\
\hline Diet & 2 & 1.364 & 0.682 & 383.59 & $<0.0001$ \\
\hline Lake $\times$ Diet & 16 & 0.147 & 0.009 & 5.17 & $<0.0001$ \\
\hline Total model & 53 & 4.020 & 0.076 & 42.66 & $<0.0001$ \\
\hline Error & 1,002 & 1.782 & 0.002 & & \\
\hline \multicolumn{6}{|l|}{$\% \mathrm{P}$} \\
\hline Lake & 8 & 1.607 & 0.201 & 16.48 & $<0.0001$ \\
\hline Genotype (lake) & 27 & 2.305 & 0.085 & 7.00 & $<0.0001$ \\
\hline Diet & 2 & 9.593 & 4.797 & 393.60 & $<0.0001$ \\
\hline Lake $\times$ Diet & 16 & 0.480 & 0.030 & 2.46 & 0.0014 \\
\hline Total model & 53 & 13.980 & 0.264 & 21.64 & $<0.0001$ \\
\hline Error & 422 & 5.143 & 0.012 & & \\
\hline \multicolumn{6}{|l|}{ PUE } \\
\hline$\overline{\text { Lake }}$ & 8 & 0.522 & 0.065 & 63.21 & $<0.0001$ \\
\hline Genotype (lake) & 27 & 0.322 & 0.012 & 11.54 & $<0.0001$ \\
\hline Diet & 2 & 0.014 & 0.007 & 6.85 & 0.0012 \\
\hline Lake $\times$ Diet & 16 & 0.065 & 0.004 & 3.95 & $<0.0001$ \\
\hline Total model & 53 & 0.920 & 0.017 & 16.80 & $<0.0001$ \\
\hline Error & 434 & 0.448 & 0.001 & & \\
\hline
\end{tabular}

Notes: Degrees of freedom $(d f)$, type III sum of squares (SS), mean squares (MS), $F$-ratio of mean squares $(F)$, and $P$-values are reported for ANOVAs examining differences in daphnid mass specific growth rate (MSGR), body $\% \mathrm{P}$ content, and $\mathrm{P}$ use efficiency (PUE).

response to dietary nutrient heterogeneity in natural environments.

Daphnia trait expression varied significantly among field-caught genotypes. As most lakes were inhabited by single species, trait variation within lakes could be attributed to either plastic responses of individual clones or intraspecific genetic differences. This phenotypic flexibility could be beneficial in lake environments where variation in diet quality can frequently occur (Kreeger et al. 1997) and might increase organismal fitness by allowing individuals to integrate elemental resources and maximize their growth rates in variable nutritional environments (Hood and Sterner 2010). In support of this hypothesis, we saw that trait expression varied among genotypes originating from different lakes due to $\mathrm{L} \times \mathrm{D}$ interactions suggesting that adaptation to lake environmental conditions (i.e., dietary $\mathrm{P}$ supply) might potentially be an important factor driving consumer phenotypic evolution at local scales (Frisch et al. 2014). Furthermore, it is also possible that these interactions may also affect larger-scale ecological processes such as regional species sorting, as $D$. pulicaria from lower nutrient lakes showed consistently higher PUE and D. mendotae, found exclusively in higher $\mathrm{P}$ lakes, displayed greater average body $\% \mathrm{P}$. Though more work including reciprocal transplant experiments is clearly needed to test whether dietary $\mathrm{P}$ supply is the main factor controlling daphnid trait differences and species distributions in these lakes, our observations contribute to a growing body of research suggesting that food quality can profoundly shape consumer growth responses and alter the elemental composition of organisms in natural lake environments (Elser et al. 2000b, Tessier and Woodruff 2002, DeMott et al. 2004, Frisch et al. 2014). But in order to better understand how these trait differences might evolve in field environments, we must first examine how genetic variation and diet combine to influence daphnid trait expression.

We documented diverse responses of individual genotypes raised across food quality gradients, providing evidence that both genetic variation and phenotypic plasticity contributed to the extensive trait variation observed in our study. Daphnia assemblages contained genetic variation for all traits, which caused significant differences in consumer trait means within diet treatments in all but one trait (i.e., D. mendotae $\% \mathrm{P}$ ). We also saw that food quality affected daphnid growth and body $\% \mathrm{P}$ resulting in plastic responses across $\mathrm{P}$ gradients, which have been well documented in these organisms (DeMott et al. 2004, Jeyasingh 2007). In addition to these main effects, we found significant $G \times D$ interactions for MSGR and PUE, indicating that variation in consumer life-history and metabolic responses that are closely tied to organismal body $\mathrm{P}$ content was also present in our study lakes (Frisch et al. 2014). Thus, within multi-clonal lake assemblages, daphnid trait expression and evolution is regulated by complex interactions between genetic variation, food quality and other sources of environmental selection, and by differences in phenotypic responses to food quality among individual clones (i.e., $\mathrm{G} \times \mathrm{D}$ interactions).

The relative strength of dietary and genetic effects on daphnid body $\% \mathrm{P}$ and on the expression of P-linked traits differed considerably. In general, differences in body $\mathrm{P}$ content were mostly caused by dietary $\mathrm{P}$ supply as elemental plasticity explained $>60 \%$ of the variation in Daphnia elemental composition in our study. These responses, while extensive, are nevertheless uninheritable by definition (Pigliucci 2001), and we detected only minor $(2-4 \%)$ amounts of heritable genetic variation in daphnid body $\% \mathrm{P}$ across diet treatments. This suggests that although flexible body stoichiometry has apparently evolved in our study lakes, there is little standing genetic variation in daphnid body $\% \mathrm{P}$ remaining for further plasticity to evolve in the absence of mutation or immigration (Vai and Lande 1985, Hill 2010). Instead, it is more plausible that selection acting on P-linked traits could alter daphnid body $\% \mathrm{P}$ as we found strong genetic effects and $\mathrm{G} \times \mathrm{D}$ interactions for MSGR and PUE.

Our results are broadly consistent with well-established relationships between Daphnia life-history and body $\mathrm{P}$ content. Similar to studies examining specieslevel differences in daphnid growth and sensitivity to $\mathrm{P}$ limitation (Seidendorf et al. 2010, Hood and Sterner 2014), growth responses differed considerably among 
TABLE 3. Effects of genotype, diet, and genotype $\times$ diet interactions $(G \times D)$ on Daphnia phenotypic trait variation.

\begin{tabular}{|c|c|c|c|c|c|c|c|c|}
\hline Species & Trait & Source & $d f$ & SS & MS & $F$ & $P$-value & $\% \operatorname{Var}$ \\
\hline \multirow[t]{15}{*}{ D. pulicaria } & \multirow[t]{5}{*}{ MSGR } & Genotype & 24 & 1.306 & 0.054 & 5.48 & $<0.0001$ & 26.51 \\
\hline & & Diet & 2 & 1.129 & 0.565 & 56.84 & $<0.0001$ & 40.02 \\
\hline & & GX D & 48 & 0.477 & 0.010 & 9.92 & $<0.0001$ & 15.89 \\
\hline & & Total Model & 74 & 2.924 & 0.040 & 39.46 & $<0.0001$ & 82.43 \\
\hline & & Error & 662 & 0.663 & 0.001 & & & 17.57 \\
\hline & \multirow[t]{5}{*}{$\% \mathrm{P}$} & Genotype & 24 & 2.554 & 0.106 & 6.64 & $<0.0001$ & 13.76 \\
\hline & & Diet & 2 & 7.011 & 3.506 & 218.69 & $<0.0001$ & 62.29 \\
\hline & & GXD & 48 & 0.769 & 0.016 & 1.50 & $0.0243^{\mathrm{ns}}$ & 2.34 \\
\hline & & Total Model & 74 & 10.649 & 0.144 & 13.47 & $<0.0001$ & 78.39 \\
\hline & & Error & 276 & 2.948 & 0.011 & & & 21.61 \\
\hline & \multirow[t]{5}{*}{ PUE } & Genotype & 24 & 0.394 & 0.016 & 4.46 & 0.0167 & 39.01 \\
\hline & & Diet & 2 & 0.025 & 0.012 & 3.32 & $0.0446^{\mathrm{ns}}$ & 3.10 \\
\hline & & GX D & 48 & 0.177 & 0.004 & 5.19 & $<0.0001$ & 27.40 \\
\hline & & Total Model & 74 & 0.592 & 0.008 & 11.26 & $<0.0001$ & 69.51 \\
\hline & & Error & 278 & 0.198 & 0.001 & & & 30.49 \\
\hline \multirow{15}{*}{ D. mendotae } & \multirow[t]{5}{*}{ MSGR } & Genotype & 10 & 0.687 & 0.069 & 9.65 & $<0.0001$ & 32.29 \\
\hline & & Diet & 2 & 0.366 & 0.183 & 25.74 & $<0.0001$ & 26.17 \\
\hline & & GXD & 20 & 0.142 & 0.007 & 3.36 & $<0.0001$ & 8.27 \\
\hline & & Total Model & 32 & 1.206 & 0.038 & 17.77 & $<0.0001$ & 66.73 \\
\hline & & Error & 286 & 0.607 & 0.002 & & & 33.27 \\
\hline & \multirow[t]{5}{*}{$\% \mathrm{P}$} & Genotype & 10 & 0.445 & 0.045 & 2.12 & $0.0733^{\mathrm{ns}}$ & 3.92 \\
\hline & & Diet & 2 & 2.630 & 1.315 & 62.52 & $<0.0001$ & 63.78 \\
\hline & & GX D & 20 & 0.421 & 0.021 & 1.35 & $0.1688^{\mathrm{ns}}$ & 3.72 \\
\hline & & Total Model & 32 & 3.500 & 0.109 & 7.00 & $<0.0001$ & 71.42 \\
\hline & & Error & 93 & 1.452 & 0.016 & & & 28.58 \\
\hline & \multirow[t]{5}{*}{ PUE } & Genotype & 10 & 0.186 & 0.019 & 6.26 & 0.0003 & 51.64 \\
\hline & & Diet & 2 & 0.001 & 0.000 & 0.12 & $0.8876^{\mathrm{ns}}$ & 0.00 \\
\hline & & GX D & 20 & 0.059 & 0.003 & 3.92 & $<0.0001$ & 19.14 \\
\hline & & Total Model & 32 & 0.255 & 0.008 & 10.54 & $<0.0001$ & 70.78 \\
\hline & & Error & 102 & 0.077 & 0.001 & & & 29.22 \\
\hline
\end{tabular}

Notes: Degrees of freedom $(d f)$, type III sum of squares (SS), mean squares (MS), $F$-ratio of mean squares $(F)$, and $P$-values are reported for ANOVAs examining differences in daphnid mass specific growth rate (MSGR), body \% $\mathrm{P}$ content, and $\mathrm{P}$ use efficiency (PUE). The proportion of trait variation (\%Var) explained by each factor is also reported from variance component analyses. Non-significant effects (ns) are indicated for $P$ values adjusted using Bonferroni corrections $(0.05 / 3=0.017)$.

genotypes fed across dietary P gradients. We also documented positive relationships between MSGR and body $\% \mathrm{P}$ within a species and with both species combined meaning that selection acting on growth could explain interspecific differences in $\mathrm{P}$ content. However, there was limited evidence of simple linear tradeoffs between growth rate and body $\% \mathrm{P}$ in our study animals as correlations between growth and body $\mathrm{P}$ within species were rather weak due to the highly variable responses of individual clones. This variation in daphnid responses to $\mathrm{P}$ limitation can partly be attributed to genetic differences in daphnid PUE. Specifically, variation in P investment into growth might explain why predicted stoichiometric trade-offs between maximum growth rate and sensitivity to dietary $\mathrm{P}$ limitation are not always observed in species adapted to different environmental conditions (DeMott and Pape 2005, Hood and Sterner 2014). This idea is supported by recent work examining evolutionary shifts in Daphnia PUE where clones evolving under chronically low P conditions showed higher PUE and muted growth responses to increased dietary $\mathrm{P}$ compared to clones from higher nutrient environments, which were much less efficient and displayed higher growth plasticity (Frisch et al. 2014). As PUE is connected to biomass production and many different aspects of $\mathrm{P}$ physiology, it is possible that evolution of PUE may help to "fine-tune" daphnid metabolism and growth with respect to their nutritional environment. If so, these changes could cause significant variation in organismal elemental composition both within and among species, which should be more generally appreciated by stoichiometric theory going forward

Overall, our study challenges widely held assumptions of invariable consumer elemental composition and of simple consistent relationships between dietary nutrition, life-history trait expression, and body \%P. We documented the potential for extensive variation in consumer MSGR and body P content among genetically distinct clones from natural assemblages. While it is unclear whether such high levels of intraspecific 


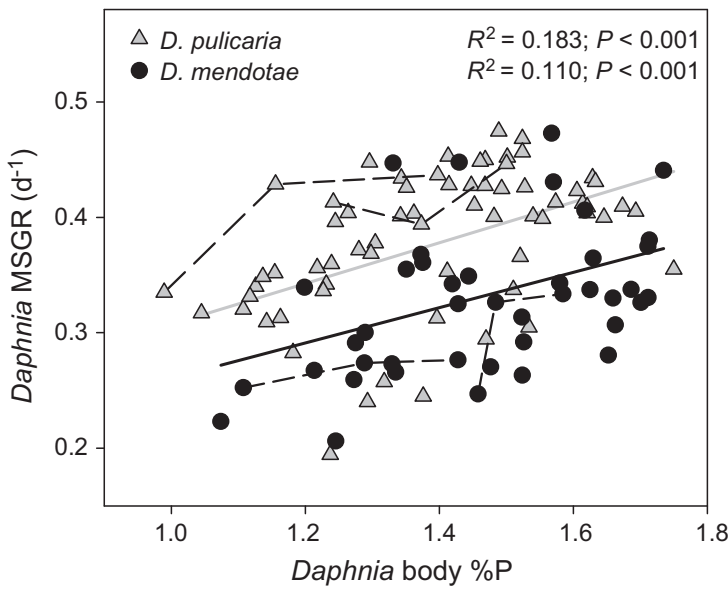

FIG. 3. Differences in the relationship between Daphnia mass specific growth rate (MSGR) and body phosphorus content $(\% \mathrm{P})$ between species and selected individual genotypes. The $R^{2}$ and $P$-values are reported from ordinary least squares regressions $(D$. pulicaria solid gray line, $D$. mendotae solid black line). Dotted lines show examples of variation in relationships between MSGR and body $\% \mathrm{P}$ for individual genotypes consuming different quality diets (low $\mathrm{P} \rightarrow$ high $\mathrm{P}$ ).

variation are common in natural populations, we found evidence that trait responses differed considerably among organisms collected across environmental $\mathrm{P}$ gradients. Thus, adaptive consumer life history responses to food quality should be more explicitly considered as a potential evolutionary mechanism shaping organismal body stoichiometry (El-Sabaawi et al. 2014, Frisch et al. 2014, Jeyasingh et al. 2014). Furthermore, the ecological effects of consumer elemental plasticity, which have traditionally been overlooked, should be better integrated into existing stoichiometric theory as intra-specific variation in a majority of our study lakes was either near or exceeded plasticity thresholds that can alter stoichiometric predictions of population and ecosystem-level dynamics (Wang et al. 2012). The addition of greater amounts of genetic variation and the incorporation of nonlinear relationships between consumer growth and body $\mathrm{P}$ content into existing stoichiometric models (e.g., Yamamichi et al. 2015) in particular may be interesting avenues of future theoretical work. In conclusion, our study has helped to outline the phenotypic envelope of two common daphnid species, but there is still a need to determine why differences in trait expression occur among clones and lake assemblages and to understand how elemental use efficiency influences the evolution of consumer elemental composition in variable nutrient environments.

\section{ACKNOWLedgments}

We thank Andrew Scott, Alice Yang, Blake Shaw, Charlotte Narr, Colleen Middleton, Elizabeth Fennell, Andrea Conine, and Mallory Nadon for lab and field help. We also thank Teresa Crease, Hailey Hunter, Jenna Dale, Shirley French, and Bill
Nelson for help and guidance with Daphnia genotyping. The manuscript was greatly improved by suggestions from Rana El-Sabaawi and by discussions with participants of the Conference of Biological Stoichiometry 2015. This work was supported by a NSERC Discovery Grant to P. C. Frost and by OGS scholarships to C. Prater and N. D. Wagner. Author contributions: C. Prater, N. D. Wagner, and P. C. Frost conceived and designed the experiments; N. D. Wagner and C. Prater conducted the experiments; C. Prater and P. C. Frost analyzed the data and wrote the manuscript.

\section{Literature Cited}

Acharya, K., M. Kyle, and J. J. Elser. 2004. Biological stoichiometry of Daphnia growth: An ecophysiological test of the growth rate hypothesis. Limnology and Oceanography 49: 656-665.

Andersen, T., and D. O. Hessen. 1991. Carbon, nitrogen, and phosphorus content of freshwater zooplankton. Limnology and Oceanography 36:807-814.

Chowdhury, P. R., and P. D. Jeyasingh. 2016. Differences in phosphorus use between ancient and extant Daphnia genotypes alters algal stoichiometry and abundance. Inland Waters 6:165-172.

Crins, W. J., P. A. Gray, P. W. C. Uhlig, and M. C. Wester. 2009. The ecosystems of Ontario, Part 1: ecozones and ecoregions. Ontario Ministry of Natural Resources, Peterborough, Ontario: Inventory, Monitoring and Assessment SIB TER IMA TR-01. Peterborough, Ontario, Canada.

Declerck, S. A. J., A. R. Malo, S. Diehl, D. Waasdorp, K. D. Lemmen, K. Proios, and S. Papakostas. 2015. Rapid adaptation of herbivore consumers to nutrient limitation: Eco-evolutionary feedbacks to population demography and resource control. Ecology Letters 18:553-562.

DeMott, W. R., and B. J. Pape. 2005. Stoichiometry in an ecological context: Testing for links between Daphnia $\mathrm{P}$-content, growth rate and habitat preference. Oecologia 142: $20-27$.

DeMott, W. R., B. J. Pape, and A. J. Tessier. 2004. Patterns and sources of variation in Daphnia phosphorus content in nature. Aquatic Ecology 38:433-440.

El-Sabaawi, R. W., J. Travis, E. Zandona, P. B. Mcintyre, D. N. Reznick, and A. Flecker. 2014. Intraspecific variability modulates interspecific variability in animal organismal stoichiometry. Ecology and Evolution 4:1505-1515.

Elser, J. J. 2006. Biological stoichiometry: a chemical bridge between ecosystem ecology and evolutionary biology. American Naturalist 168(Suppl):S25-S35.

Elser, J. J., D. R. Dobberfuhl, N. A. MacKay, and J. H. Schampel. 1996. Organism size, life history, and N: P stoichiometry: Towards a unified view of cellular and ecosystem processes. BioScience 46:674-684.

Elser, J. J., et al. 2000a. Nutritional constraints in terrestrial and freshwater food webs. Nature 408:578-580.

Elser, J. J., W. J. O'Brien, D. R. Dobberfuhl, and T. E. Dowling. $2000 b$. The evolution of ecosystem processes: Growth rate and elemental stoichiometry of a key herbivore in temperate and arctic habitats. Journal of Evolutionary Biology 13: 845-853.

Elser, J. J., et al. 2003. Growth rate-stoichiometry couplings in diverse biota. Ecology Letters 6:936-943.

Falster, D. S., D. I. Warton, and I. J. Wright. 2006. SMATR: Standardised major axis tests and routines, ver 2.0. https:// github.com/dfalster/smatr/.

Frisch, D., P. K. Morton, P. R. Chowdhury, B. W. Culver, J. K. Colbourne, L. J. Weider, and P. D. Jeyasingh. 2014. 
A millennial-scale chronicle of evolutionary responses to cultural eutrophication in Daphnia. Ecology Letters 17: 360-368.

Frost, P. C., M. A. Evans-White, Z. V. Finkel, T. C. Jensen, and V. Matzek. 2005. Are you what you eat? Physiological constraints on organismal stoichiometry in an elementally imbalanced world. Oikos 109:18-28.

Frost, P. C., J. P. Benstead, W. F. Cross, H. Hillebrand, J. H. Larson, M. A. Xenopoulos, and T. Yoshida. 2006. Threshold elemental ratios of carbon and phosphorus in aquatic consumers. Ecology Letters 9:774-779.

Gibert, J. P., A. I. Dell, J. P. DeLong, and S. Pawar. 2015. Scaling-up trait variation from individuals to ecosystems. Advances in ecological research. First edition. Elsevier, Amsterdam, The Netherlands.

Gorokhova, E., T. E. Dowling, L. J. Weider, T. J. Crease, and J. J. Elser. 2002. Functional and ecological significance of rDNA intergenic spacer variation in a clonal organism under divergent selection for production rate. Proceedings of the Royal Society B 269:2373-2379.

Greenberg, A. E., L. S. Clesceri, and A. D. Eaton. 1992. APHA Method 3500-MG: Standard Methods for the Examination of Water and Wastewater, Eighteenth edition. American Public Health Association, Washington DC.

Haney, J. F., et al. 2013. An Image-based key to the zooplankton of North America v. 5.0. http://cfb.unh.edu/cfbkey/html/ index.html.

Hebert, P. D. N., and M. J. Beaton. 1993. Methodologies for allozyme analysis using cellulose acetate electrophoresis. Helena Laboratories, Beaumont, Texas, USA.

Hicks, A. L., and P. C. Frost. 2011. Shifts in aquatic macrophyte abundance and community composition in cottage developed lakes of the Canadian Shield. Aquatic Botany 94: 9-16.

Hill, W. G. 2010. Understanding and using quantitative genetic variation. Philosophical Transactions of the Royal Society B 365:73-85.

Hood, J. M., and R. W. Sterner. 2010. Diet mixing: do animals integrate growth or resources across temporal heterogeneity? American Naturalist 176:651-663.

Hood, J. M., and R. W. Sterner. 2014. Carbon and phosphorus linkages in Daphnia growth are determined by growth rate, not species or diet. Functional Ecology 28:1156-1165.

Jeyasingh, P. D. 2007. Plasticity in metabolic allometry: The role of dietary stoichiometry. Ecology Letters 10:282-289.

Jeyasingh, P. D., L. J. Weider, and R. W. Sterner. 2009. Genetically-based trade-offs in response to stoichiometric food quality influence competition in a keystone aquatic herbivore. Ecology Letters 12:1229-1237.

Jeyasingh, P. D., R. D. Cothran, and M. Tobler. 2014. Testing the ecological consequences of evolutionary change using elements. Ecology and Evolution 4:528-538.

Kawecki, T. J., and D. Ebert. 2004. Conceptual issues in local adaptation. Ecology Letters 7:1225-1241.

Kilham, S. S., D. A. Kreeger, S. G. Lynn, C. E. Goulden, and L. Herrera. 1998. COMBO: a defined freshwater culture medium for algae and zooplankton. Hydrobiologia 377: $147-159$.
Kreeger, D. A., C. E. Goulden, S. S. Kilham, S. G. Lynn, S. Datta, and S. J. Interlandi. 1997. Seasonal changes in the biochemistry of Lake Seston. Freshwater Biology 38:539-554.

Littell, R. C., W. W. Stroup, and R. J. Freund. 2002. SAS for linear models. Fourth edition. SAS Institute, Cary, North Carolina, USA.

Matthews, B., et al. 2011. Toward an integration of evolutionary biology and ecosystem science. Ecology Letters 14:690-701.

Miner, B. G., S. E. Sultan, S. G. Morgan, D. K. Padilla, and R. A. Relyea. 2005. Ecological consequences of phenotypic plasticity. Trends in Ecology and Evolution 20:685-692.

Persson, J., P. Fink, A. Goto, J. M. Hood, J. Jonas, and S. Kato. 2010. To be or not to be what you eat: Regulation of stoichiometric homeostasis among autotrophs and heterotrophs. Oikos 119:741-751.

Pigliucci, M. 2001. Phenotypic plasticity: beyond nature and nurture. Johns Hopkins University Press, Baltimore, Maryland, USA

Pigliucci, M. 2005. Evolution of phenotypic plasticity: Where are we going now? Trends in Ecology and Evolution 20: 481-486.

Schlichting, C. D., and M. Pigliucci. 1998. Phenotypic evolution: a reaction norm perspective. Sinauer Associates, Sunderland, Massachusetts, USA.

Searle, S. R., G. Casella, and C. E. McCulloch. 1992. Variance components. Page book. First edition. John Wiley and Sons, Hoboken, New Jersey, USA.

Seidendorf, B., N. Meier, A. Petrusek, M. Boersma, B. Streit, and K. Schwenk. 2010. Sensitivity of Daphnia species to phosphorus-deficient diets. Oecologia 162:349-357.

Stearns, S. C. 1992. The evolution of life histories. Oxford University Press, Oxford, UK

Sterner, R. W., D. D. Hagemeier, W. L. Smith, R. F. Smith, L. Smith, and F. Smith. 1993. Phytoplankton nutrient limitation and food quality for Daphnia. Limnology and Oceanography $38: 857-871$.

Tessier, A. J., and C. E. Goulden. 1987. Cladoceran juvenile growth. Limnology and Oceanography 32:680-686.

Tessier, A. J., and P. Woodruff. 2002. Trading off the ability to exploit rich versus poor food quality. Ecology Letters 5: 685-692.

Vai, S., and R. Lande. 1985. Genotype-environment interaction and the evolution of phenotypic plasticity. Evolution 39: 505-522.

Wagner, N. D., and P. C. Frost. 2012. Responses of alkaline phosphatase activity in Daphnia to poor nutrition. Oecologia $170: 1-10$

Wang, H., R. W. Sterner, and J. J. Elser. 2012. On the "strict homeostasis" assumption in ecological stoichiometry. Ecological Modelling 243:81-88.

Weider, L. J., P. D. Jeyasingh, and K. G. Looper. 2008. Stoichiometric differences in food quality: Impacts on genetic diversity and the coexistence of aquatic herbivores in a Daphnia hybrid complex. Oecologia 158:47-55.

Yamamichi, M., C. L. Meunier, A. Peace, C. Prater, and M. A. Rúa. 2015. Rapid evolution of a consumer stoichiometric trait destabilizes consumer-producer dynamics. Oikos 124: 960-969.

\section{SUPPORTING INFORMATION}

Additional supporting information may be found in the online version of this article at http://onlinelibrary.wiley.com/doi/ 10.1002/ecy.1795/suppinfo 